\title{
Clinical profiles of patients with surgically resected pheochromocytoma and paraganglioma
}

Sun Hwa Lee ${ }^{1}$, Jae-Hyeong Park² ${ }^{2}$ Ja-Yeon Lee ${ }^{1}$, Sang-Rok Lee ${ }^{1}$, Kyoung-Suk Rhee ${ }^{1}$, Jei-Keon Chae ${ }^{1}$, Won-Ho Kim ${ }^{1}$, Ji-Young Sul ${ }^{3}$, Jin Kyung $\mathrm{Oh}^{2}$, Hee-Jin Kwon ${ }^{2}$, Jae-Hwan Lee ${ }^{2}$, and In-Whan Seong ${ }^{2}$

${ }^{1}$ Division of Cardiology, Department of Internal Medicine, Chonbuk National University Medical School, Research Institute of Clinical Medicine of Chonbuk National University-Biomedical Research Institute of Chonbuk National University Hospital, Jeonju; ${ }^{2}$ Division of Cardiology, Department of Internal Medicine, Chungnam National University College of Medicine, Regional Cardiocerebrovascular Center, Chungnam National University Hospital, Daejeon; ${ }^{3}$ Department of Surgery, Chungnam National University Hospital, Daejeon, Korea

Received: June 28, 2018 Revised : September 13, 2018 Accepted: November 11, 2018

\section{Correspondence to}

Jae-Hyeong Park, M.D.

Division of Cardiology,

Department of Internal

Medicine, Chungnam National

University Hospital, 282

Munhwa-ro, Jung-gu, Daejeon

35015 , Korea

Tel: +82-42-28o-7167

Fax: +82-42-28o-8238

E-mail:jaehpark@cnu.ac.kr
Background/Aims: Pheochromocytoma and paraganglioma (PPGL) are catecholamine-producing tumors that can cause blood pressure (BP) elevation and cardiovascular complications. Clinical presentation of these tumors may be changed through widespread use of imaging studies, which enables detection of PPGLs before onset of symptoms. We investigated clinical profiles of patients with surgically resected PPGLs.

Methods: From 2005 to 2017 , 111 consecutive patients with surgically resected PPGLs in two tertiary hospitals in Korea were studied.

Results: Mean age was $52 \pm 16$ years, 57 patients (51.4\%) were male and 54 (48.6\%) were hypertensive. Twenty-nine PPGLs $(26.1 \%)$ were extra-adrenal paragangliomas. Sixteen (14.4\%) and seven patients (6.3\%) (Group 1, n = 23) were diagnosed during work-up of hypertension and transient cardiomyopathy respectively, and the remainder (Group 2, $\mathrm{n}=88$ ) were incidentalomas detected during routine abdominal imaging. Patients in the Group 1 were younger and more frequently symptomatic, and had higher BPs, heart rates and levels of urinary catecholamines than those in the Group 2. Paragangliomas were less frequent and secretion of epinephrine and metanephrine was more predominant in the Group 1 than in Group 2. After the surgical resections, $18.2 \%$ of patients still needed antihypertensive medications.

Conclusions: Out of 111 patients with surgically resected PPGLs, 88 (79.3\%) were diagnosed as incidentalomas. Seven patients presented with transient cardiomyopathy and 16 with hypertension. Tumor location and secretion of catecholamine may vary depending on the presence of symptoms.

Keywords: Pheochromocytoma; Paraganglioma; Catecholamines; Hypertension; Cardiomyopathies

\section{INTRODUCTION}

Pheochromocytoma and paraganglioma (PPGL) are neuroendocrine tumors from chromaffin cells and usually originated from the medulla of the adrenal gland or extra-adrenal location in which chromaffin cells are located $[1,2]$. PPGLs release catecholamines into circu- lation, and these can be related to episodic headache, dizziness and palpitation as results of stimulation of beta-adrenergic receptors. Because alpha-adrenergic stimulation can cause elevation of blood pressure (BP), PPGLs are considered one of causes of secondary hypertension. Moreover, excessive catecholamine release increases heart rate and can provoke transient cardio- 
myopathy (CMP) associated with catecholamine [3,4]. However, diagnosis of PPGLs is quite challenging because their clinical manifestations are nonspecific and variable, and they are rare tumors. Since widespread use of computed tomography (CT) scans, especially for the purpose of health screening, has led to increased rate of detection of adrenal tumors without symptoms (incidentalomas), the prevalence of clinical presentations suggesting catecholamine excess might have changed from past studies in patients with PPGLs. Thus, we evaluated the clinical presentations of patients with surgically resected PPGLs.

\section{METHODS}

\section{Study population}

All consecutive patients with PPGLs which were surgically resected and confirmed by pathologic examination were retrospectively enrolled from January 2005 to December 2017 in two tertiary teaching hospitals in Korea (Chungnam National University Hospital [CNNUH] and Chonbuk National University Hospital [CBNUH]).

\section{Study measures}

The baseline demographic characteristics including age, sex, past history of hypertension and antihypertensive medication, clinical symptoms, levels of urinary and plasma catecholamines, and radiologic findings including CT were investigated with the review of medical records. Excretion of 24-hour urinary catecholamines and levels of plasma catecholamines were measured by a liquid chromatographic method (reference, 24-hour urinary norepinephrine $<80 \mu \mathrm{g} /$ day, epinephrine $<22$ $\mu \mathrm{g} /$ day, vanillyl mandelic acid [VMA] $<8 \mathrm{mg} /$ day, metanephrine $<0.8 \mathrm{mg} /$ day, plasma free metanephrine $<0.50$ $\mathrm{nmol} / \mathrm{L}$, normetanephrine $<0.90 \mathrm{nmol} / \mathrm{L}$, epinephrine 1 to $20 \mathrm{pg} / \mathrm{mL}$, and norepinephrine 15 to $80 \mathrm{pg} / \mathrm{mL}$ ) [5] . Catecholamine secretion was considered to be significantly increased if the 24-hour urinary excretion of metanephrine, epinephrine or norepinephrine, or plasma levels of metanephrine or normetanephrine was five times higher than their cut-off values.

Electrocardiographic (ECG) findings were classified into four categories: left ventricular hypertrophy by voltage criteria, significant ST-T abnormalities, nonspecific
ST-T changes, and normal ECG. Presence of CMP was defined as echocardiographic evidence of left ventricular dysfunction and regional wall motion abnormalities (RWMA) inconsistent with coronary territories or transient RWMA with no evidence of obstructive coronary lesions. The tumor with the presence of local invasion into surrounding organs and tissues or distant metastases was considered to have malignant potential. This study protocol was approved by Institutional Review Board of each hospital (IRB No. CNNUH, 2016-12-047; CBNUH, 2018-07-002) and written informed consent was waived because the study was retrospective and anonymous clinical data were used for analysis.

\section{Statistical analysis}

Data are expressed as mean \pm standard deviation for continuous variables and as frequencies (percentages) for the categorical variables. Statistical analysis was performed using SPSS version 22.0 (IBM Inc., Chicago, IL, USA) software. A Mann-Whitney U test and Student's $t$ test were performed for statistical comparison between the numeric data, while a chi-square test was performed for the categorical data. Statistical significance was determined as a $p$ value $<0.05$.

\section{RESULTS}

\section{Clinical characteristics of overall patients with PPGLs}

During the study period, a total of 111 patients with PPGLs were enrolled. Mean age was $52 \pm 16$ years (range, 14 to 81 ) and 57 patients (51.4\%) were male. Mean systolic and diastolic BPs of overall patients were 133.2 \pm 23.3 and $80.4 \pm 15.0 \mathrm{mmHg}$, respectively. Fifty-four patients (48.6\%) had a history of hypertension and 48 (45.3\%) were taking $1.9 \pm 0.8$ antihypertensive drugs. Their mean BP at presentation was $137.3 \pm 27.7 / 81.9 \pm 16.6 \mathrm{mmHg}$.

ECG was normal in 78 patients (71.6\%). Left ventricular hypertrophy by voltage criteria was noted in 22 patients (20.2\%), significant ST-T abnormalities in seven (6.4\%) and nonspecific ST-T changes in two (1.8\%). Echocardiography was performed in 46 patients (41.8\%) due to cardiovascular symptoms such as chest pain or dyspnea (n $=8)$, abnormal ECG $(n=14)$ or cardiovascular risk factors $(n=8)$, and for preoperative screening $(n=16)$. Mean left ventricular ejection fraction (LVEF) was $58.9 \% \pm 10.0 \%$. 
Table 1. Clinical characteristics of patients with surgically resected pheochromocytoma and paraganglioma at initial presentation and after surgery according to their clinical presentations

\begin{tabular}{|c|c|c|c|c|}
\hline Variable & Total $(\mathrm{n}=111)$ & Group $1(n=23)$ & Group $2(n=88)$ & $p$ value \\
\hline \multicolumn{5}{|l|}{ Baseline characteristics } \\
\hline Age, yr & $52 \pm 16$ & $44 \pm 16$ & $54 \pm 15$ & 0.005 \\
\hline Male sex & $57(51.4)$ & $9(39.1)$ & $48(54 \cdot 5)$ & 0.243 \\
\hline History of hypertension & $54(48.6)$ & $12(52.2)$ & $42(47 \cdot 7)$ & 0.816 \\
\hline Antihypertensive medications & $48(45 \cdot 3)$ & $14(60.9)$ & $34(41.0)$ & 0.090 \\
\hline No. of pills & $1.9 \pm 0.8$ & $1.9 \pm 0.6$ & $1.9 \pm 0.9$ & 0.753 \\
\hline Diabetes & $20(18.0)$ & $6(26.1)$ & $14(15 \cdot 9)$ & 0.359 \\
\hline Dyslipidemia & $11(9.9)$ & $3(13.0)$ & $8(9.1)$ & 0.695 \\
\hline \multicolumn{5}{|l|}{ Vital signs at presentation } \\
\hline $\mathrm{SBP}, \mathrm{mmHg}$ & $133.2 \pm 23.3$ & $147 \cdot 5 \pm 28.3$ & $129.6 \pm 20.6$ & 0.012 \\
\hline $\mathrm{DBP}, \mathrm{mmHg}$ & $80.4 \pm 15.0$ & $93.1 \pm 20.6$ & $77 \cdot 3 \pm 11.3$ & 0.002 \\
\hline HR, bpm & $78.0 \pm 12.9$ & $84.4 \pm 12.0$ & $76.5 \pm 12.8$ & 0.014 \\
\hline \multicolumn{5}{|l|}{ Symptoms } \\
\hline Headache & $18(16.2)$ & $12(52.2)$ & $6(6.8)$ & $<0.001$ \\
\hline Dizziness & $12(10.8)$ & $5(21.7)$ & $7(8.0)$ & 0.123 \\
\hline Chest pain & $13(11.7)$ & $9(39.1)$ & $4(4 \cdot 5)$ & $<0.001$ \\
\hline Palpitation & $15(13 \cdot 5)$ & $8(34.8)$ & $7(8.0)$ & 0.003 \\
\hline \multicolumn{5}{|l|}{ Postoperative clinical course } \\
\hline $\mathrm{SBP}, \mathrm{mmHg}$ & $123.3 \pm 13.3$ & $123.0 \pm 11.2$ & $123 \cdot 4 \pm 13 \cdot 9$ & 0.905 \\
\hline $\mathrm{DBP}, \mathrm{mmHg}$ & $75 \cdot 0 \pm 8.3$ & $76.8 \pm 7.6$ & $74.5 \pm 8.4$ & 0.277 \\
\hline $\mathrm{HR}, \mathrm{bpm}$ & $76.0 \pm 12.4$ & $82.3 \pm 12.9$ & $74 \cdot 5 \pm 11.8$ & 0.015 \\
\hline Antihypertensive medications & $20(18.2)$ & $6(26.1)$ & $14(16.1)$ & 0.269 \\
\hline No. of pills & $1.6 \pm 0.9$ & $1.2 \pm 0.4$ & $1.8 \pm 0.9$ & 0.074 \\
\hline
\end{tabular}

Values are presented as mean \pm SD or number (\%).

SBP, systolic blood pressure; DBP, diastolic blood pressure; HR, heart rate.

Eighty-two patients (73.9\%) had pheochromocytomas and 29 (26.1\%) of PPGLs was extra-adrenal paragangliomas in the head and neck, chest, abdomen, and pelvis (middle ear, carotid body, aorta, retroperitoneum, duodenum, bladder, and scrotum). Twenty PPGLs (18.0\%) showed malignant potential and four patients (3.6\%) had metastatic lesions. After the surgery, 20 patients (18.2\%) still needed $1.6 \pm 0.9$ antihypertensive medications and their BP was $124.8 \pm 15.0 / 74.4 \pm 7.1 \mathrm{mmHg}$.

\section{Comparison of symptomatic PPGLs versus inciden- talomas}

In 88 patients (79.3\%), PPGLs were incidentally found during routine abdominal imaging. Other reasons of work-up for PPGLs were paroxysmal elevation of BP with clinical suspicion of secondary hypertension in 16 patients (14.4\%) and transient CMP in seven patients (6.3\%). We divided our study patients into two groups; 23 patients with clinical presentations of BP elevation or CMP were classified into Group 1 (suspected group) and the remainders (87 patients) who were diagnosed incidentally into Group 2 (incidental group). Their clinical characteristics and laboratory findings are listed in Tables 1 and 2.

Patients in the Group 1 were significantly younger than those in the Group 2 ( $44 \pm 16$ years old vs. $54 \pm 15$ years old, $p=0.005)$. Clinical symptoms related to catecholamine excess such as headache $(52.2 \%$ vs. $6.8 \%, p<$ 
Table 2. Laboratory, electrocardiographic, and echocardiographic findings of patients with surgically resected pheochromocytoma and paraganglioma and their characteristics according to their clinical presentations

\begin{tabular}{|c|c|c|c|c|}
\hline Variable & Total $(\mathrm{n}=111)$ & Group $1(n=23)$ & Group $2(n=88)$ & $p$ value \\
\hline \multicolumn{5}{|l|}{ Characteristics of tumors } \\
\hline Location & & & & 0.033 \\
\hline Adrenal (pheochromocytoma) & $82(73.9)$ & $21(91.3)$ & $61(69 \cdot 3)$ & \\
\hline Extra-adrenal (paraganglioma) & $29(26.1)$ & $2(8.7)$ & $27(30.7)$ & \\
\hline Maximal diameter (mm) in imaging studies & $43.6 \pm 31.0$ & $41.9 \pm 15.7$ & $44.1 \pm 33.8$ & 0.778 \\
\hline Presence of necrosis & $35(31.5)$ & $10(43.5)$ & $25(28.4)$ & 0.196 \\
\hline Malignant potential & $20(18.2)$ & $4(17 \cdot 4)$ & $16(18.4)$ & 0.912 \\
\hline Metastatic lesions & $4(3.6)$ & $1(4 \cdot 3)$ & $3(3 \cdot 4)$ & 0.838 \\
\hline \multicolumn{5}{|l|}{ Laboratory findings } \\
\hline BUN, mg/dL & $14.9 \pm 6.1$ & $15.6 \pm 6.9$ & $14.0 \pm 5.8$ & 0.281 \\
\hline Serum creatinine, mg/dL & $0.9 \pm 1.1$ & $0.8 \pm 0.3$ & $0.9 \pm 1.2$ & 0.613 \\
\hline Total cholesterol, mg/dL & $190.5 \pm 40.8$ & $201.0 \pm 42.9$ & $187.1 \pm 39.8$ & 0.154 \\
\hline Urine metanephrine (<o.8), mg/day & $123.1 \pm 707.3$ & $302.2 \pm 1,238.5$ & $61.3 \pm 387.0$ & 0.002 \\
\hline Urine epinephrine (1.7-2.2), $\mu \mathrm{g} / \mathrm{day}$ & $118.6 \pm 327 \cdot 9$ & $320.4 \pm 586.5$ & $43 \cdot 5 \pm 66.1$ & 0.019 \\
\hline Urine norepinephrine (12.1-80), $\mu \mathrm{g} /$ day & $274.5 \pm 339 \cdot 7$ & $292.8 \pm 385.0$ & $267.7 \pm 334.6$ & 0.693 \\
\hline Urine $\operatorname{VMA}(<8), \mathrm{mg} /$ day & $19 \cdot 7 \pm 33 \cdot 3$ & $25 \cdot 3 \pm 26.0$ & $17 \cdot 9 \pm 35 \cdot 3$ & 0.007 \\
\hline Plasma epinephrine (1-20), pg/mL & $189.4 \pm 381.8$ & $219 \cdot 4 \pm 237.1$ & $178.3 \pm 428.1$ & 0.813 \\
\hline Plasma norepinephrine ( $15-80), \mathrm{pg} / \mathrm{mL}$ & $1,106.6 \pm 1,558.4$ & $1,442.8 \pm 1,775.6$ & $975.1 \pm 1,500.4$ & 0.512 \\
\hline Plasma metanephrine (< 0.5$), \mathrm{nmol} / \mathrm{L}$ & $1.52 \pm 1.41$ & $2.28 \pm 1.57$ & $1.13 \pm 1.25$ & 0.198 \\
\hline Plasma normetanephrine (< o.9), nmol/L & $6.31 \pm 6.37$ & $4.64 \pm 4.48$ & $7.14 \pm 7.27$ & 0.547 \\
\hline \multicolumn{5}{|l|}{ Secretion of catecholamine } \\
\hline Norepinephrine & $14(38.9)$ & $4(50)$ & $10(35 \cdot 7)$ & 0.465 \\
\hline Epinephrine & $56(65 \cdot 1)$ & $18(85 \cdot 7)$ & $38(58.5)$ & 0.023 \\
\hline Non-secreting & $8(22.9)$ & $1(12.5)$ & $7(25 \cdot 9)$ & 0.427 \\
\hline Electrocardiographic findings & & & & 0.003 \\
\hline Normal & $78(71.6)$ & $15(65.2)$ & $63(73 \cdot 3)$ & \\
\hline LVH by voltage criteria & $22(20.2)$ & $2(8.7)$ & $20(23 \cdot 3)$ & \\
\hline Significant ST-T abnormalities & $7(6.4)$ & $5(21.7)$ & $2(2.3)$ & \\
\hline Nonspecific ST-T changes & $2(1.8)$ & $1(4 \cdot 3)$ & $1(1.2)$ & \\
\hline \multicolumn{5}{|l|}{ Echocardiographic findings } \\
\hline Study performed & $46(41.8)$ & $16(69.6)$ & $30(34 \cdot 5)$ & 0.002 \\
\hline LVEF, \% & $58.9 \pm 10.0$ & $54 \cdot 3 \pm 14 \cdot 4$ & $61.0 \pm 6.2$ & 0.114 \\
\hline
\end{tabular}

Values are presented as number (\%) or mean \pm SD.

BUN, blood urea nitrogen; VMA, vanillyl mandelic acid; LVH, left ventricular hypertrophy; LVEF, left ventricular ejection fraction.

0.001 ), chest pain (39.1\% vs. $4.5 \%, p<0.001$ ), and palpitation (34.8\% vs. $8.0 \%, p=0.003$ ) were more frequent in the Group 1 than in the Group 2. In the Group 1, systolic $(147.5 \pm 28.3 \mathrm{mmHg}$ vs. $129.6 \pm 20.6 \mathrm{mmHg}, p=0.012)$ and diastolic $\mathrm{BP}(93.1 \pm 20.6 \mathrm{mmHg}$ vs. $77.3 \pm 11.3 \mathrm{mmHg}, p=$
$0.002)$, and heart rate $(84.4 \pm 12.0 \mathrm{bpm}$ vs. $76.5 \pm 12.8 \mathrm{bpm}$, $p=0.014)$ were significantly higher than those in the Group 2. In terms of location, extra-adrenal paragangliomas were significantly less frequent in the Group 1 than the Group 2 (8.7\% vs. 30.7\%, $p=0.033$ ) although 
the size of the tumor, malignant potential, presence of necrosis, and distant metastasis were comparable in the two groups (Table 2). The levels of urinary excretion of metanephrine, epinephrine and VMA were significantly higher and increased secretion of epinephrine was more predominant ( $85.7 \%$ vs. $58.5 \%, p=0.023$ ) in Group 1 than in Group 2 (Table 2). Patients in Group 1 showed significant ST-T abnormalities on ECG more frequently than those in Group 2 (21.7\% vs. 2.3\%, $p=0.003$ ).

\section{Clinical profiles of patients with catecholamine CMP}

The clinical profiles of patients with CMP are summarized in Table 3. The main symptom was chest pain. ST change and T-wave abnormalities were commonly found in the ECG examinations. Apical ballooning was found in four patients and inverted Takotsubo pattern (basal ballooning) was demonstrated in three patients in the echocardiographic studies. All patients with CMP recovered within 12 days (range, 3 to 12). One patient had cardiogenic shock and was successfully treated with extracorporeal membrane oxygenation for 12 days and continuous renal replacement therapy for 25 days (Fig. 1).

The clinical characteristics of patients presenting with $\operatorname{CMP}(\mathrm{n}=7)$ and those without CMP $(\mathrm{n}=104)$ are shown in Table 4. All tumors in patients with CMP were pheochromocytomas and their secretion of epinephrine was significantly increased. Patients with CMP had more frequent symptoms, history of hypertension, and higher diastolic BP than those without CMP, and showed trends of more frequent diabetes and dyslipidemia, poorer LVEF and higher need for antihypertensives after surgery (Table 4).

\section{DISCUSSION}

In this study, we examined 111 patients with surgically resected PPGLs and demonstrated that the majority (79.3\%) of patients were diagnosed incidentally and 23 patients $(20.7 \%)$ were diagnosed during work-up for other causes; 16 (14.4\%) for evaluation of BP elevation and seven (6.3\%) for work-up of CMP. In patients presented with hypertension or CMP, adrenal locations, increased secretion of epinephrine, and cardiovascular symptoms were more prominent than in those with incidentaloma.

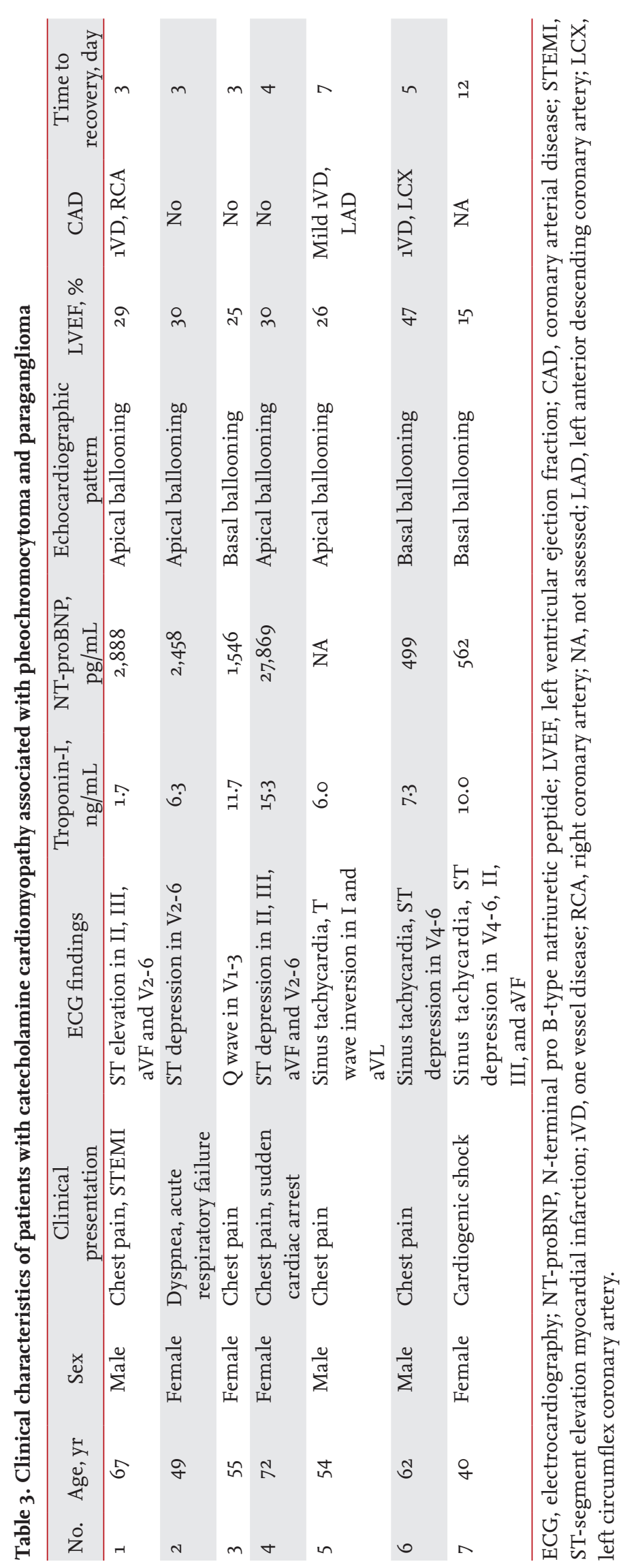




\section{KJIM ${ }^{-}$}
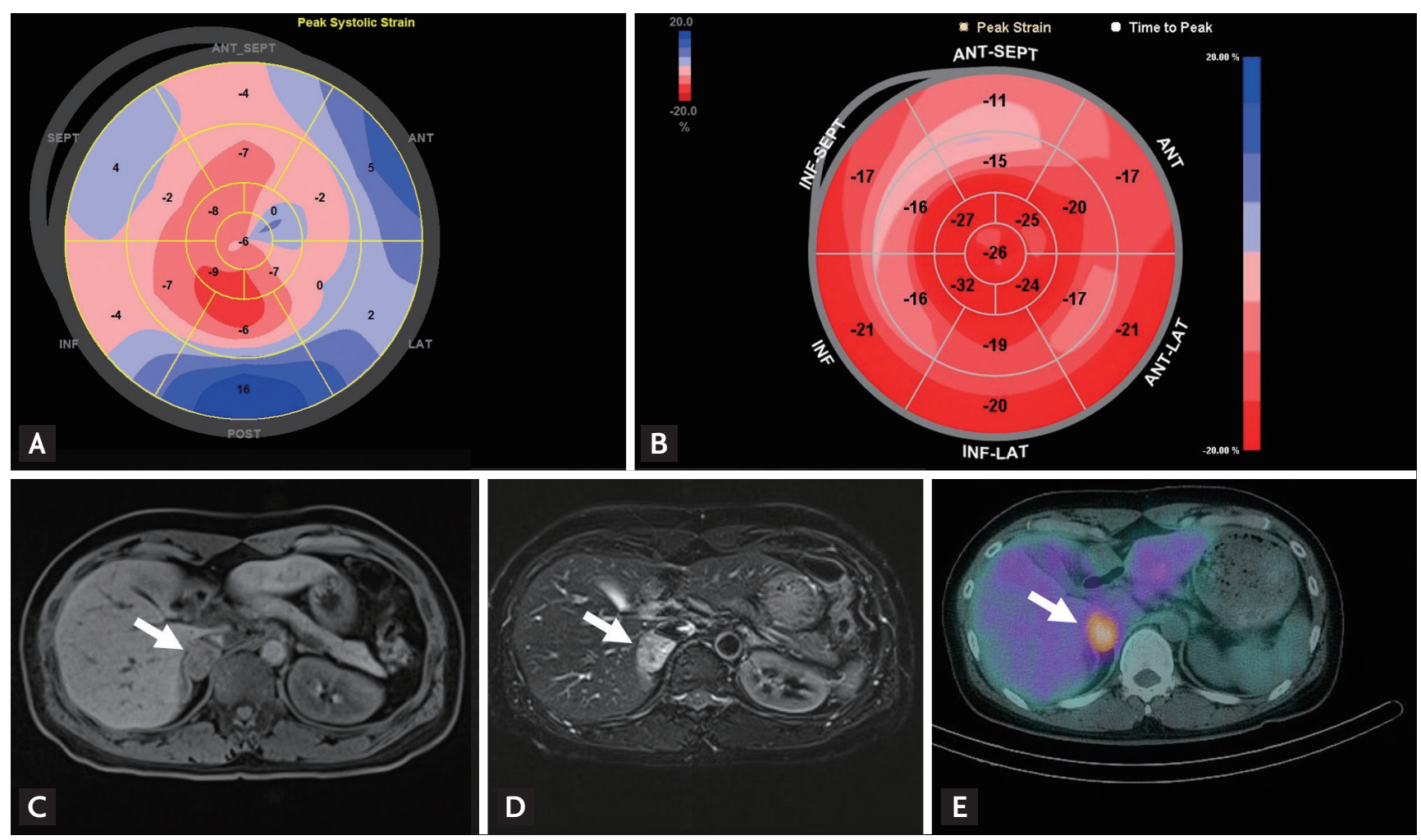

Figure 1. Representative case. A 40-year-old woman (Case no. 7) presented with acute dyspnea after dilation and curettage due to missed abortion. Her blood pressure was $80 / 50 \mathrm{mmHg}$ and heart rate was $120 \mathrm{bpm}$. (A) Two-dimensional echocardiography on admission day showed basal dyskinesis with severely depressed left ventricular ejection fraction (LVEF) of $15 \%$ and global longitudinal strain (GLS) of $-1.9 \%$. (B) Two months after treatment with mechanical ventilation, extracorporeal membrane oxygenation and continuous renal replacement therapy, her LVEF was improved to 61\% and GLS to -21.0\%. Her urinary excretions of metanephrine and epinephrine were increased to $2.0 \mathrm{mg} /$ day and $50.9 \mu \mathrm{g} /$ day, respectively. Abdominal magnetic resonance imaging study showed a $2.8 \times 2.4 \mathrm{~cm}$-sized mass (arrows) at right suprarenal area with (C) low on T1-weighted and (D) high signal intensity on T2-weighted images. (E) ${ }^{123}$ I-metaiodobenzylguanidine (MIBG) single-photon emission computed tomography demonstrated intense MIBG uptake in the right adrenal mass (arrow) which was compatible with pheochromocytoma. ANT, anterior wall; SEPT, septum; INF, inferior wall; LAT, lateral wall; POST, posterior wall.

PPGLs are catecholamine-producing tumors originated from chromaffin cells in the adrenal glands or along the extra-adrenal sympathetic ganglia. Typical symptoms associated with the secretion of catecholamine include headache, tachycardia, dizziness, and sweating [6]. In our study, the frequency of symptoms associated with PPGLs and the levels of urinary excretion of catecholamines were significantly higher in the Group 1 than in the Group 2. These findings suggest that their symptoms were associated with the excess of catecholamines.

The reported incidence of hypertension is about $80 \%$ to $90 \%$ of patients with pheochromocytoma and half of them demonstrate sustained hypertension $[7,8]$. However, recent popular use of CT imaging can increase the proportion of asymptomatic patients [9] and about $5 \%$ to $6 \%$ of adrenal incidentalomas have been reported to be PPGLs $[10,11]$. Therefore, hypertension can be absent in a substantial portion of patients with PPGLs $[12,13]$. A German study [12] including 201 patients diagnosed with PPGL between 1973 and 2007 showed that only $10 \%$ of cases presented the typical triad of symptoms; $29.4 \%$ of them were incidentally detected and incidentalomas were increasing in recent years. In our study, PPGLs were clinically suspected as a secondary cause of hypertension in only $14.4 \%$ of patients with PPGLs because of paroxysmal elevation of BP. Imaging studies done during routine health screening were the most common route of detection of PPGLs in this study. The incidence of hypertension was $48.6 \%$ and comparable in both groups. Out of 54 patients with a medical history of 
Table 4. Comparison of patients presented with catecholamine cardiomyopathy and those without cardiomyopathy associated with pheochromocytoma and paraganglioma

\begin{tabular}{|c|c|c|c|}
\hline Variable & $\operatorname{SCM}(n=7)$ & Non-SCM $(n=104)$ & $p$ value \\
\hline Age, yr & $57 \pm 11$ & $51 \pm 16$ & 0.364 \\
\hline Male sex & $3(42.9)$ & $53(51.5)$ & 0.660 \\
\hline Hypertension & $6(85.7)$ & $47(45 \cdot 7)$ & 0.040 \\
\hline Diabetes & $3(42.9)$ & $17(16.5)$ & 0.080 \\
\hline Dyslipidemia & $2(28.6)$ & $9(8.7)$ & 0.091 \\
\hline \multicolumn{4}{|l|}{ At presentation } \\
\hline Headache & $2(28.6)$ & $15(14.6)$ & 0.321 \\
\hline Dizziness & 0 & $12(11.7)$ & 0.339 \\
\hline Chest pain & $6(85.7)$ & $7(6.8)$ & $<0.001$ \\
\hline Palpitation & $3(42.9)$ & $12(11.7)$ & 0.020 \\
\hline $\mathrm{SBP}, \mathrm{mmHg}$ & $144 \cdot 7 \pm 19 \cdot 7$ & $132.4 \pm 23.4$ & 0.177 \\
\hline $\mathrm{DBP}, \mathrm{mmHg}$ & $91.6 \pm 18.2$ & $79.6 \pm 14.5$ & 0.041 \\
\hline Paraganglioma & o & $29(28.2)$ & 0.102 \\
\hline Maximal diameter, mm & $44.9 \pm 15 \cdot 4$ & $43.5 \pm 31.9$ & 0.909 \\
\hline \multicolumn{4}{|l|}{ Secretion of catecholamine } \\
\hline Norepinephrine & $1(50)$ & $13(38.2)$ & 0.740 \\
\hline Epinephrine & $7(100)$ & $49(62.0)$ & 0.043 \\
\hline Non-secreting & o & $8(24.2)$ & 0.428 \\
\hline Urine metanephrine (< o.8), mg/day & $8.4 \pm 5 \cdot 3$ & $120.9 \pm 702.7$ & 0.675 \\
\hline Urine epinephrine (1.7-2.2), $\mu \mathrm{g} /$ day & $534.8 \pm 790.2$ & $65.9 \pm 186.3$ & 0.206 \\
\hline Urine norepinephrine (12.1-280), $\mu \mathrm{g} /$ day & $68.0 \pm 40.7$ & $261.0 \pm 327.8$ & 0.422 \\
\hline Urine VMA $(<8), \mathrm{mg} /$ day & $38.8 \pm 39.6$ & $17 \cdot 9 \pm 31.2$ & 0.126 \\
\hline Plasma metanephrine (< 0.5$), \mathrm{nmol} / \mathrm{L}$ & $2.69 \pm 2.17$ & $1.28 \pm 1.24$ & 0.214 \\
\hline Plasma normetanephrine (<0.9), nmol/L & $2.98 \pm 2.48$ & $6.98 \pm 6.78$ & 0.444 \\
\hline Echocardiography performed & $7(100)$ & $39(37.9)$ & 0.001 \\
\hline LVEF, \% & $47 \cdot 0 \pm 17 \cdot 6$ & $61.1 \pm 5.9$ & 0.079 \\
\hline \multicolumn{4}{|l|}{ After surgery } \\
\hline Antihypertensive medications & $4(57 \cdot 1)$ & $16(15 \cdot 5)$ & 0.006 \\
\hline $\mathrm{SBP}, \mathrm{mmHg}$ & $128.0 \pm 11.4$ & $123.1 \pm 13.4$ & 0.424 \\
\hline $\mathrm{DBP}, \mathrm{mmHg}$ & $77.0 \pm 5.1$ & $74.9 \pm 8.4$ & 0.579 \\
\hline
\end{tabular}

Values are presented as mean $\pm \mathrm{SD}$ or number (\%).

SCM, stress-induced cardiomyopathy; SBP, systolic blood pressure; DBP, diastolic blood pressure; VMA, vanillyl mandelic acid; LVEF, left ventricular ejection fraction.

hypertension, 34 (63.0\%) discontinued antihypertensive medication after the resection of the PPGLs (eight in the Group 1 and 20 in the Group 2). Meticulous history taking, application of imaging studies and biochemical screening can lead to early diagnosis of PPGL as a cause of secondary hypertension.

Another interesting finding of our study is that tran- sient CMP was an initial presentation of PPGLs. The socalled catecholamine CMP presents transient, reversible cardiac dysfunction. Shaw et al. [14] reported echocardiographic findings of transient CMP in a patient with pheochromocytoma in 1987. Catecholamine CMP can demonstrate typical transient apical ballooning [15,16] or inverted Takotsubo-type CMP (transient mid- or bas- 
al ventricular ballooning) [4,17]. Catecholamines can induce myocardial damage by microvascular dysfunction of the coronary arteries, multivessel epicardial spasm, impaired fatty acid metabolism, myocarditis and catecholamine-mediated myocardial dysfunction [17,18]. Although a majority of patients with catecholamine CMP recover within several days, catecholamine toxicity may produce long-lasting myocardial alterations even after curative resection of PPGLs [19]. The incidence of transient CMP was 6.3\% in our study. All patients were clinically improved within 12 days after the onset of symptoms. One of our patients with CMP presented as a profound cardiogenic shock with basal ballooning requiring mechanical circulatory support (Fig. 1).

About $15 \%$ to $20 \%$ of PPGLs are derived from extra-adrenal chromaffin tissue referred to as paragangliomas and are known to not produce catecholamine [20]. In our study, $26.1 \%$ of the PPGLs were extra-adrenal. The prevalence of paragangliomas was lower in Group 1 than in Group 2 (8.7\% vs. 30.7\%, $p=0.033$ ) and all tumors associated with CMP were pheochromocytomas.

It has been suggested that predominant catecholamine can determine clinical manifestations of PPGLs $[21,22]$. Increased plasma epinephrine has been reported to be associated with paroxysmal elevation of BP and norepinephrine with sustained hypertension [21]. We demonstrated that the secretion of epinephrine and metanephrine was found to be significantly increased in patients in Group 1 compared to Group 2, although the levels were far above the upper normal values in both groups. This finding was more evident in patients with CMP, which were consistent with epinephrine-related toxic CMP. On the other hand, in patients without CMP, urinary excretion of norepinephrine and the level of plasma free normetanephrine were higher than in patients with CMP, although the rates did not reach statistical significance due to the small number of patients. This can partly be explained biochemically; extra-adrenal PPGLs are predominantly associated with secretion of norepinephrine and normetanephrine [10].

Our study has several limitations. First, this study is a retrospective study through a review of medical records. Therefore, symptoms associated with catecholamine excess were not adequately described and not all levels of urinary and plasma catecholamines were measured. Because echocardiographic studies were performed in the selected patients with suspected cardiac dysfunction, the incidence and patterns of CMP could not be assessed exactly. PPGLs are very rare tumors and can be missed, especially when there are no apparent symptoms. To evaluate the exact incidence and pattern of the clinical presentations associated with PPGLs, a prospective cohort study will be needed.

\section{KEY MESSAGE}

1. Out of a total of 111 patients with surgically resected pheochromocytoma and paraganglioma (PPGL), seven (6.3\%) presented with transient cardiomyopathy (CMP) and 16 (14.4\%) with elevated blood pressure.

2. Along with hypertension, transient CMP can be an initial presentation of PPGL.

3. Location of tumor and secretion of catecholamine of may vary depending on the presence of symptoms in patients with PPGL.

\section{Conflict of interest}

No potential conflict of interest relevant to this article was reported.

\section{REFERENCES}

1. Sheps SG, Jiang NS, Klee GG, van Heerden JA. Recent developments in the diagnosis and treatment of pheochromocytoma. Mayo Clin Proc 1990;65:88-95.

2. Lam KY, Lo CY. Composite pheochromocytoma-ganglioneuroma of the adrenal gland: an uncommon entity with distinctive clinicopathologic features. Endocr Pathol 1999;10:343-352.

3. Bybee KA, Prasad A. Stress-related cardiomyopathy syndromes. Circulation 2008;118:397-409.

4. Kim EM, Park JH, Park YS, et al. Catecholamines may play an important role in the pathogenesis of transient mid- and basal ventricular ballooning syndrome. J Korean Med Sci 2008;23:898-902.

5. Gerlo EA, Sevens C. Urinary and plasma catecholamines and urinary catecholamine metabolites in pheochromocytoma: diagnostic value in 19 cases. Clin Chem 1994;40:250256. 
6. Stein PP, Black HR. A simplified diagnostic approach to pheochromocytoma. A review of the literature and report of one institution's experience. Medicine (Baltimore) 1991;70:46-66.

7. Omura M, Saito J, Yamaguchi K, Kakuta Y, Nishikawa T. Prospective study on the prevalence of secondary hypertension among hypertensive patients visiting a general outpatient clinic in Japan. Hypertens Res 2004;27:193-202.

8. Zelinka T, Eisenhofer G, Pacak K. Pheochromocytoma as a catecholamine producing tumor: implications for clinical practice. Stress 2007;10:195-203.

9. Amar L, Servais A, Gimenez-Roqueplo AP, Zinzindohoue F, Chatellier G, Plouin PF. Year of diagnosis, features at presentation, and risk of recurrence in patients with pheochromocytoma or secreting paraganglioma. J Clin Endocrinol Metab 2005;90:2110-2116.

10. van Berkel A, Lenders JW, Timmers HJ. Diagnosis of endocrine disease: biochemical diagnosis of phaeochromocytoma and paraganglioma. Eur J Endocrinol 2014;170:R109-R119.

11. Ahn SH, Kim JH, Baek SH, et al. Characteristics of adrenal incidentalomas in a large, prospective computed tomography-based multicenter study: the COAR Study in Korea. Yonsei Med J 2018;59:501-510.

12. Kopetschke R, Slisko M, Kilisli A, et al. Frequent incidental discovery of phaeochromocytoma: data from a German cohort of 201 phaeochromocytoma. Eur J Endocrinol 2009;161:355-361.

13. Noshiro T, Shimizu K, Watanabe T, et al. Changes in clinical features and long-term prognosis in patients with pheochromocytoma. Am J Hypertens 2000;13:35-43.

14. Shaw TR, Rafferty P, Tait GW. Transient shock and myocardial impairment caused by phaeochromocytoma cri- sis. Br Heart J 1987;57:194-198.

15. Wood R, Commerford PJ, Rose AG, Tooke A. Reversible catecholamine-induced cardiomyopathy. Am Heart J 1991;121:610-613.

16. Takizawa M, Kobayakawa N, Uozumi H, et al. A case of transient left ventricular ballooning with pheochromocytoma, supporting pathogenetic role of catecholamines in stress-induced cardiomyopathy or takotsubo cardiomyopathy. Int J Cardiol 2007;114:e15-e17.

17. Hurst RT, Askew JW, Reuss CS, et al. Transient midventricular ballooning syndrome: a new variant. J Am Coll Cardiol 2006;48:579-583.

18. Tsuchihashi K, Ueshima K, Uchida T, et al. Transient left ventricular apical ballooning without coronary artery stenosis: a novel heart syndrome mimicking acute myocardial infarction. Angina Pectoris-Myocardial Infarction Investigations in Japan. J Am Coll Cardiol 2001;38:11-18.

19. Ferreira VM, Marcelino M, Piechnik SK, et al. Pheochromocytoma is characterized by catecholamine-mediated myocarditis, focal and diffuse myocardial fibrosis, and myocardial dysfunction. J Am Coll Cardiol 2016;67:23642374 .

20. Lenders JW, Eisenhofer G, Mannelli M, Pacak K. Phaeochromocytoma. Lancet 2005;366:665-675.

21. Ito Y, Fujimoto Y, Obara T. The role of epinephrine, norepinephrine, and dopamine in blood pressure disturbances in patients with pheochromocytoma. World J Surg 1992;16:759-763.

22. Olson SW, Deal LE, Piesman M. Epinephrine-secreting pheochromocytoma presenting with cardiogenic shock and profound hypocalcemia. Ann Intern Med 2004;140:849-851. 